\title{
Reproductive and metabolic features during puberty in sons of women with polycystic ovary syndrome
}

\section{Nicolás Crisosto', Bárbara Echiburú1, Manuel Maliqueo', Marta Luchsinger1, Pedro Rojas ${ }^{2}$, Sergio Recabarren ${ }^{2}$ and Teresa Sir-Petermann'}

${ }^{1}$ Endocrinology and Metabolism Laboratory, West Division, School of Medicine, University of Chile, Santiago, Chile ${ }^{2}$ Laboratory of Animal Physiology and Endocrinology, Faculty of Veterinary Sciences, University of Concepción, Chillán, Chile
Endocrine CONNECTIONS

\begin{abstract}
Context: Intrauterine life may be implicated in the origin of polycystic ovary syndrome (PCOS) modifying the endocrine and metabolic functions of children born to PCOS mothers independently of the genetic inheritance and gender. The aim of the present study was to evaluate the reproductive and metabolic functions in sons of women with PCOS during puberty.

Methods: Sixty-nine PCOS sons (PCOSs) and 84 control sons of 7-18 years old matched by the Tanner stage score were studied. A complete physical examination was conducted including anthropometric measurements (weight, height, waist, hip and body mass index). An oral glucose tolerance test was performed and circulating concentrations of luteinizing hormone, follicle-stimulating hormone (FSH), sex hormone-binding globulin, testosterone, androstenedione (A4), 17 $\alpha$-hydroxyprogesterone (17-OHP) and AMH were determined in the fasting sample.

Results: Waist-to-hip ratio, FSH and androstenedione levels were significantly higher in the PCOSs group compared to control boys during the Tanner stage II-III. In Tanner stages II-III and IV-V, PCOSs showed significantly higher total cholesterol and LDL levels. Propensity score analysis showed that higher LDL levels were attributable to the PCOSs condition and not to other metabolic factors. AMH levels were comparable during all stages. The rest of the parameters were comparable between both groups.

Conclusions: Sons of women with PCOS show increased total cholesterol and LDL levels during puberty, which may represent latent insulin resistance. Thus, this is a group that should be followed and studied looking for further features of insulin resistance and cardiovascular risk markers. Reproductive markers, on the other hand, are very similar to controls.
\end{abstract}

Correspondence should be addressed to T Sir-Petermann

Email

tsir@med.uchile.cl
Endocrine Connections (2017) 6, 607-613

\section{Introduction}

Polycystic ovary syndrome (PCOS) is a common familial endocrine-metabolic disorder that affects women of reproductive age, characterized by irregular menses, chronic anovulation, infertility and hyperandrogenism
$(1,2)$. A genetic cause of the syndrome was suggested many years ago (3), and phenotypic and family aggregation studies have demonstrated that a significant number of female relatives of PCOS patients are affected
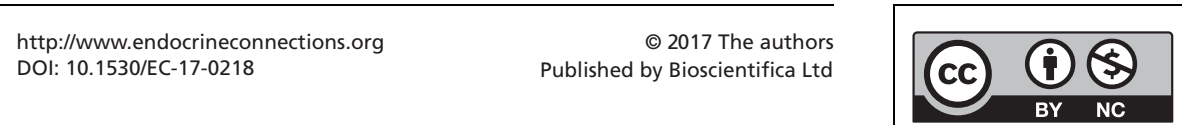

This work is licensed under a Creative Commons Attribution-NonCommercial 4.0 International License. 
with the condition $(4,5,6,7,8)$. Moreover, it has been proposed that intrauterine life, as an environmental factor, is implicated in the origin of $\operatorname{PCOS}(8,9,10,11)$ and may modify the endocrine and metabolic functions of a child born to a PCOS mother independently of the genetic inheritance and gender $(12,13,14)$. Therefore, it is possible to suggest that both daughters and sons born to PCOS mothers could be affected showing reproductive and metabolic abnormalities similar to those observed in women with PCOS.

We have studied daughters of women with PCOS during different stages of development finding several features that may represent early metabolic and reproductive markers of the syndrome and that may be modified through interventions that improve the adverse pregnancy environment present in PCOS women $(13,15)$.

On the other hand, the reproductive and metabolic features of male members of PCOS families have been less documented in the literature. Some of the phenotypes proposed include abnormalities in hair distribution, such as increased hair growth and balding or premature male balding (16). Other studies have described abnormalities in plasma luteinizing hormone (LH) levels (17) and dehydroepiandrosterone sulphate concentrations in male members of PCOS families (18). Our group reported that brothers of PCOS women show increased 17-hydroxyprogesterone levels in response to leuprolide acetate resembling those described in women with PCOS (19). In a further study, we reported that basal concentrations of gonadotropins, sex steroids and inhibin B were comparable between PCOS sons (PCOSs) and control sons (Cs) during early infancy, childhood and adulthood. We also obtained similar results in stimulated gonadotropin and sex steroid concentrations. However, AMH serum concentrations were higher in PCOSs compared with Cs during early infancy and childhood with no difference during adulthood indicating a possible early dysfunction of Sertoli cells. Nevertheless, the sperm-count analysis was similar between both groups (14). Regarding metabolic features, we observed increased weight from an early age and some indicators of insulin resistance during adulthood (12).

Puberty is a transition period between childhood and adulthood accompanied by profound changes in the body composition, insulin sensitivity and sex steroid production, and then it is a critical period in the setting of metabolic and reproductive features observed during adulthood (20).

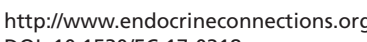

Therefore, the aim of the present study was to evaluate the reproductive and metabolic functions in sons of women with PCOS during puberty in order to analyse the transition from childhood to adulthood in this setting.

\section{Materials and methods}

We included 69 PCOSs and 84 Cs between 7 and 18 years of age. The study was designed to analyse PCOSs and controls transversally. Boys were sub-grouped into three categories according to their Tanner stage score for testis development: Tanner I, Tanner II-III and Tanner IV-V. The boys included in the study were not taking any medication. All boys were born from singleton pregnancies. Some of these boys participated in our previous studies during infancy and childhood $(12,14)$.

Mothers with PCOS were recruited from patients attending the Unit of Endocrinology and Reproductive Medicine, University of Chile, Santiago, Chile. The diagnosis of PCOS was made according to the National Institutes of Health Consensus Criteria which was the criteria used at the time of their diagnosis during reproductive age before pregnancy (21). Mothers with PCOS exhibited chronic oligomenorrhea or amenorrhea and hirsutism during reproductive age. In addition, PCOS women showed the characteristic ovarian morphology of polycystic ovaries in ultrasound, based on the criteria described by Adams and coworkers (22). These inclusion criteria for PCOS mothers were similar to those previously reported (15). As control mothers, we selected women who had a history of regular 28- to 32-day menstrual cycles, absence of hirsutism and other manifestations of hyperandrogenism and no history of infertility or pregnancy complications.

\section{Study protocol}

The boys were admitted with their mothers to our Clinical Research Centre. We performed a complete physical examination on each boy, including anthropometric measurements (weight, height, waist, hip, body mass index (BMI) and BMI standard deviation (s.D.) score (SDS) calculated using the Growth Analyser Program and the US BMI-for-age growth charts) (23). These growth curves have been shown to be applicable to contemporary Chilean population (24). Obesity was defined as a body weight $>95$ th percentile. Testis volume was determined by a single endocrinologist (N C) using the Prader orchidometer. 
In both groups of boys, an oral glucose tolerance test $(1.75 \mathrm{~g} / \mathrm{kg}$, up to a maximum of $75 \mathrm{~g}$ glucose in $250 \mathrm{~mL}$ water) after a 12-h overnight fast was performed. Blood samples $(5 \mathrm{~mL})$ were drawn before 30,60 and $120 \mathrm{~min}$ after the glucose load. Serum glucose and insulin were determined in each sample. Glucose tolerance was evaluated using the American Diabetes Association criteria (25). The homeostasis model assessment for insulin resistance (HOMA-IR) and the whole-body insulin sensitivity composite index (ISI) were calculated as previously described $(26,27)$. In addition, circulating concentrations of LH, follicle-stimulating hormone (FSH), sex hormonebinding globulin (SHBG), testosterone, androstenedione (A4), 17 $\alpha$-hydroxyprogesterone (17-OHP), AMH and lipid profile were determined in the fasting sample. Basal serum SHBG and testosterone were used to calculate the FAI as the ratio of serum testosterone/SHBG $\times 100$.

The protocol was approved by the institutional review board of the University of Chile. All parents signed informed consents, and boys gave their assent before entering the study.

\section{Assays}

Serum AMH was assayed by enzyme immunoassay (Immunotech-Beckman Coulter, Marseille, France). Analytical sensitivity was $2.1 \mathrm{pmol} / \mathrm{L}$ and intra- and interassay coefficients of variation were $5.3 \%$ and $8.7 \%$, respectively. Serum glucose and lipid profile were determined by the glucose oxidase method (Biosystem). The intraassay coefficient of variation of this method was $<1.2 \%$ and interassay was $<2.7 \%$. LDL cholesterol was calculated according to Friedewald formula.

Serum insulin was assayed by IRMA (Diasource, Nivelles, Belgium since 2010) with a sensitivity of $1.0 \mu \mathrm{IU} / \mathrm{mL}$ and intra- and interassay coefficients of variation of 3 and 7\%, respectively. Serum LH, FSH and SHBG were determined by IRMA izotop (Institute of Isotops Co. Ltd. (Izotop), Budapest, Hungary). Assay sensitivities were $0.05 \mathrm{IU} / \mathrm{mL}, 0.02 \mathrm{IU} / \mathrm{mL}$ and $0.22 \mathrm{nmol} / \mathrm{L}$, respectively. Intra- and interassay coefficients of variation were $1.0 \%$ and $3.1 \%$ for $\mathrm{LH}, 2.5 \%$ and $2.7 \%$ for FSH and $4.9 \%$ and $3.8 \%$ for SHBG, respectively.

Serum testosterone, androstenedione and 17-OHP were assayed by RIA (Diagnostic Systems Laboratories and DIASource) and the limits of detection were $0.1,0.1$ and $0.03 \mathrm{ng} / \mathrm{mL}$. The intra- and interassay coefficients of variation were 3.5 and $5.5 \%, 3.8 \%$ and $7.5 \%$ and 9.4 and $18 \%$, respectively.

\section{Statistical evaluation}

The present study was designed to analyse PCOS boys and controls transversally. The number of subjects was calculated based on our previous studies showing significant differences in LDL and insulin levels in adult PCOSs (12). Data are expressed as median and interquartile range. Data distribution was assessed by the Kolmogorov-Smirnov test showing a not normal distribution. Differences between study groups were assessed with the Mann-Whitney test. Correlations were assessed using the Spearman test adjusted for BMI and age. Statistical analysis was performed with Graph Pad Prism 6.0 package and the SPSS package version 22. A $P$ value of less than 0.05 was considered statistically significant. A propensity score analysis, using the variables age, Tanner stage, waist-to-hip ratio (WHR), z score BMI, testosterone, androstenedione, ISI score, HOMA and trygliceride levels, was used to establish if the differences observed in LDL levels were attributable only to the PCOSs condition.

\section{Results}

Table 1 shows the clinical and anthropometric characteristics of both groups classified in three sub-groups according to the Tanner stage. WHR was significantly higher in the PCOSs group during the Tanner stage II-III. There were no other anthropometric differences between control and PCOSs in none of the stages studied. Testicular volumes were similar between both groups within the different Tanner stage sub-groups.

Table 2 shows the hormonal and metabolic data in both groups classified according to the Tanner stage. During Tanner stages II-III, PCOSs showed higher FSH and androstenedione levels compared to control boys. AMH levels were comparable during all stages.

During Tanner stages II-III and IV-V, PCOSs showed significantly higher total cholesterol and LDL levels.

Propensity score analysis using the variables age, Tanner stage, WHR, $Z$ score BMI, testosterone, androstenedione, ISI score, HOMA and triglyceride levels established that there is a significant difference in LDL levels (73.03 s.D.: 4.21 vs 88.4 s.D.: $4.89 ; P=0.022$ ) between PCOSs and Cs that is attributable to the PCOSs condition.

Table 3 shows the correlations found between the different parameters in the whole group and according to the Tanner stage adjusted by BMI and age, showing a positive correlation among waist, LDL and total cholesterol with surrogates of insulin resistance.

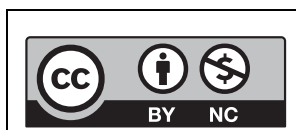

This work is licensed under a Creative Commons Attribution-NonCommercial 4.0 International License. 


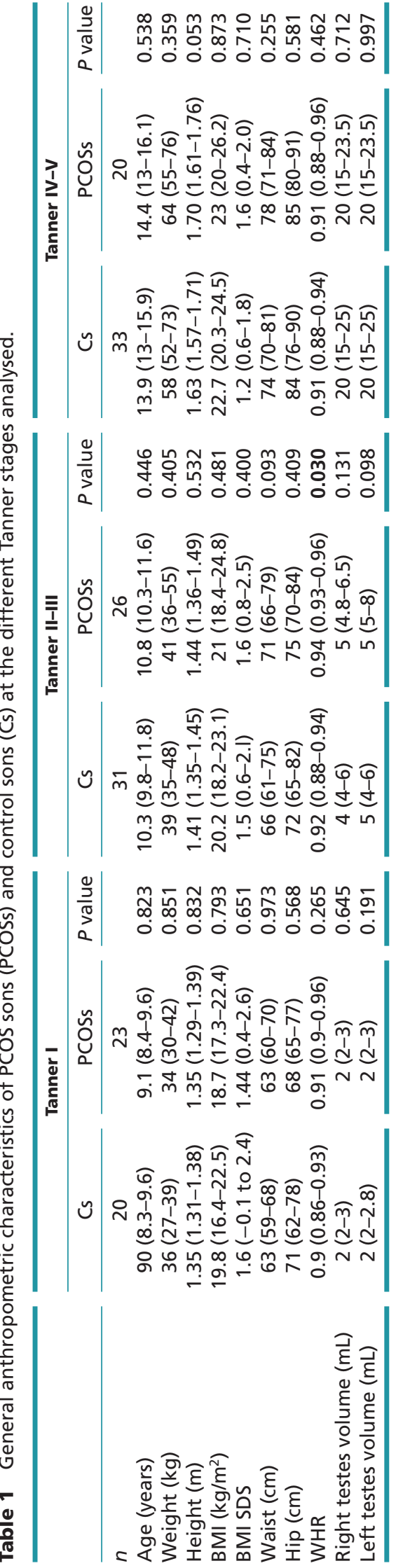

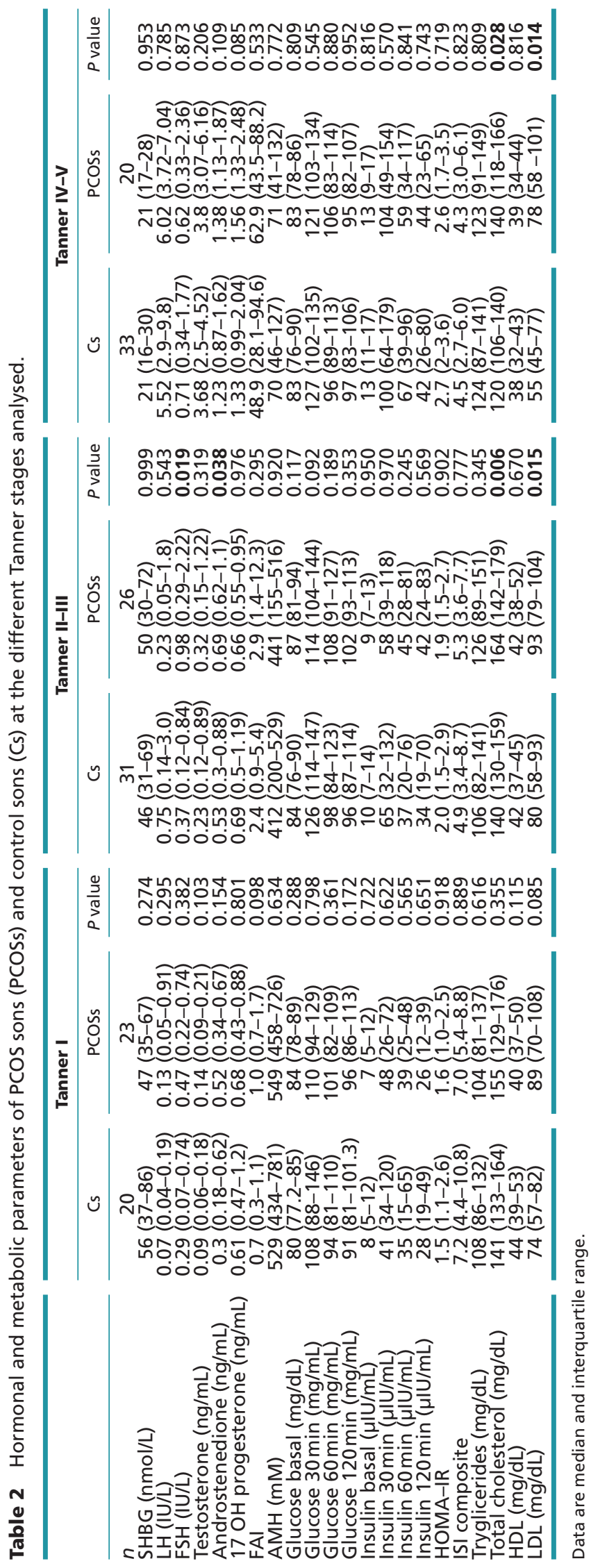

http://www.endocrineconnections.org DOI: 10.1530/EC-17-0218

() 2017 The authors Published by Bioscientifica Ltd

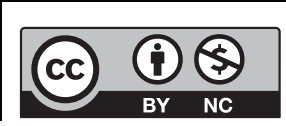

This work is licensed under a Creative Commons Attribution-NonCommercial 4.0 International License. 
Table 3 Spearman correlations adjusted by age and BMI in the whole groups.

\begin{tabular}{|c|c|c|}
\hline & $\boldsymbol{R}$ & $P$ Value \\
\hline \multicolumn{3}{|l|}{ Whole group } \\
\hline \multicolumn{3}{|l|}{ Waist } \\
\hline Basal insulin & 0.275 & 0.01 \\
\hline 30 min insulin & 0.215 & 0.01 \\
\hline HOMA-IR & 0.267 & 0.03 \\
\hline HDL & -0.210 & 0.02 \\
\hline \multicolumn{3}{|l|}{ Tanner II-III } \\
\hline \multicolumn{3}{|l|}{ Waist } \\
\hline Basal insulin & 0.342 & 0.02 \\
\hline $30 \mathrm{~min}$ insulin & 0.341 & 0.02 \\
\hline HOMA-IR & 0.317 & 0.03 \\
\hline HDL & -0.308 & 0.04 \\
\hline \multicolumn{3}{|l|}{ Total cholesterol } \\
\hline 120 min glucose & 0.330 & 0.02 \\
\hline 30 min insulin & 0.335 & 0.02 \\
\hline 60 min insulin & 0.348 & 0.02 \\
\hline \multicolumn{3}{|l|}{ LDL } \\
\hline Basal glucose & 0.331 & 0.02 \\
\hline $30 \mathrm{~min}$ insulin & 0.344 & 0.02 \\
\hline $60 \mathrm{~min}$ insulin & 0.357 & 0.01 \\
\hline Composite & -0.394 & 0.01 \\
\hline \multicolumn{3}{|l|}{ Tanner IV-V } \\
\hline \multicolumn{3}{|l|}{ Waist } \\
\hline Basal insulin & 0.398 & 0.02 \\
\hline HOMA-IR & 0.408 & 0.01 \\
\hline
\end{tabular}

\section{Discussion}

In the present study, we analysed the pubertal period of sons born to PCOS women, a stage of profound changes in the body composition, insulin sensitivity and sex steroid production. We are, thus, completing our previous observations in PCOSs during infancy, childhood and adulthood.

We found an increased WHR along with higher cholesterol and LDL levels during Tanner stages II-III. PCOSs also showed higher FSH and androstenedione levels during this stage. The cholesterol alterations were also observed during the Tanner stage IV-V but with no anthropometric differences.

Despite being comparable in age, BMI and Tanner stage, PCOSs seem to develop early derangements in cholesterol levels. We have previously reported this observation in children and adult PCOSs (12). Nevertheless, in that case, PCOSs BMI was also significantly higher and the differences disappeared when controlled by BMI. In the present study, BMIs were comparable and propensity score analysis showed that this difference was attributable to the PCOSs condition. Thus, cholesterol alterations in this group seem to represent an intrinsic independent feature probably associated with an incipient state of insulin resistance. Although insulin and glucose levels were comparable during all Tanner stages, during Tanner stages II-III, LDL was positively correlated with basal glucose, 30, 60 and average insulin levels and negatively correlated with ISI composite. Thus, indirectly LDL levels might be reflecting insulin resistance at this stage.

The increase in WHR and a trend to higher waist circumference (WC) in Tanner II-III boys may also represent a latent insulin resistance that is expressed later on during adulthood. In a larger study, waist was associated with insulin resistance during adolescence. In this regard, associations between adiposity measures, such as WC, and insulin resistance have been shown to be stronger in children during puberty compared with those who had completed pubertal development (28). In this regard, in the current study, WC was correlated with different surrogates of insulin resistance during Tanner stages II-V. Thus, this might be a good marker for metabolic disruption in pubertal boys.

Daughters of women with PCOS show increased insulin and triglyceride levels from an early stage (15). On the other hand, boys show comparable insulin and triglyceride levels but significantly higher LDL and total cholesterol levels. This feature is still present during adulthood but is accompanied by increased insulin levels (12). Thus, we can suggest that there is a sexual dimorphism regarding the expression and onset of insulin resistance in the offspring of PCOS women, with an early appearance of hypertriglyceridemia and hyperinsulinemia in girls and an early increased LDL and late hyperinsulinaemia in boys.

This sexual dimorphism has also been found in normal Chilean girls, showing higher triglyceride levels in adolescent girls compared to boys (29). Thus, the expression of insulin resistance in daughters and sons of PCOS women seems to be determined in part by these naturally occurring gender differences.

Later, during adult life, this insulin resistance is expressed as increased insulin levels and higher BMI in PCOS women and adult PCOSs as we have previously reported (12).

Opposite to what we found in younger PCOSs, in whom we observed increased AMH levels, pubertal PCOSs show normal AMH levels, same as the adult PCOSs group in our previous report (14). Thus, it seems like, despite having a period of possible delayed maturation during infancy and childhood, Sertoli cells seem to have an appropriate function during puberty. 
Starting puberty, we observed increased FSH levels and higher androstenedione levels that then normalize during the next stage of puberty. Higher androstenedione may come from the testis or the adrenal gland. A possible explanation is that there is an increased secretion of androgens from the adrenal gland as has been suggested in PCOS women $(30,31)$, and then as the testis takes over androgen secretion, this difference disappears. Higher FSH levels are harder to interpret and may reflect a slightly earlier maturation of the gonadal axis.

In conclusion, sons of women with PCOS show increased total cholesterol and LDL levels during puberty, which may represent latent insulin resistance that is later on translated into higher insulin levels and a higher BMI. Thus, this is a group that should be followed and studied looking for further features of insulin resistance and cardiovascular risk markers. Reproductive markers, on the other hand, are very similar to controls so there is no important evidence pointing at any significant reproductive difference in this group.

\section{Declaration of interest}

N C, B E, M M, H L, P R, S R and T S-P declare that there is no conflict of interest that could be perceived as prejudicing the impartiality of the research reported.

\section{Funding}

Supported by the Fondo Nacional de Desarrollo Científico y Tecnológico (National Fund for Scientific and Technological Research; Fondecyt; Grants 1030487-1151531), and the Alexander von Humboldt Foundation.

\section{Authors contribution statement}

Nicolas Crisosto: patient recruitment and evaluation, data analysis, manuscript preparation; Bárbara Echiburú: data analysis, hormonal determinations; Manuel Maliqueo: data analysis, hormonal determinations. Marta Lushsinger: hormonal determinations. Pedro Rojas: data analysis; Sergio Recabarren: data analysis; Teresa Sir-Petermann: general coordination of the project, patient recruitment and evaluation, manuscript preparation.

\section{Acknowledgement}

The authors would like to thank Gabriel Cavada for his help with the statistical analysis of this paper.

\section{References}

1 Ehrmann DA, Barnes RB \& Rosenfield RL. Polycystic ovary syndrome as a form of functional ovarian hyperandrogenism due to dysregulation of androgen secretion. Endocrine Reviews 199516 322-353. (doi:10.1210/er.16.3.322)

2 Azziz R, Woods KS, Reyna R, Key TJ, Knochenhauer ES \& Yildiz BO. The prevalence and features of the polycystic ovary syndrome in an unselected population. Journal of Clinical Endocrinology and Metabolism 200489 2745-2749. (doi:10.1210/jc.2003-032046)

3 Cooper HE, Spellacy WN, Prem KA \& Cohen WD. Hereditary factors in the Stein-Leventhal syndrome. American Journal of Obstetrics and Gynecology 1968100 371-387. (doi:10.1016/S00029378(15)33704-2)

4 Givens JR. Familial polycystic ovarian disease. Endocrinology Metabolism Clinics of North America 198817 771-783.

5 Hague WM, Adams J, Reeders ST, Peto TE \& Jacobs HS. Familial polycystic ovaries: a genetic disease? Clinical Endocrinology 198829 593-605. (doi:10.1111/j.1365-2265.1988.tb03707.x)

6 Lunde O, Magnus P, Sandvik L \& Høglo S. Familial clustering in the polycystic ovarian syndrome. Gynecologic and Obstetric Investigation 198928 23-30. (doi:10.1159/000293493)

7 Azziz R, Carmina E, Chen Z, Dunaif A, Laven JS, Legro RS, Lizneva D, Natterson-Horowtiz B, Teede HJ \& Yildiz BO. Polycystic ovary syndrome. Nature Reviews Disease Primers 2016216057. (doi:10.1038/nrdp.2016.57)

8 Puttabyatappa M, Cardoso RC \& Padmanabhan V. Effect of maternal PCOS and PCOS-like phenotype on the offspring's health. Molecular and Cellular Endocrinology 2016435 29-39. (doi:10.1016/j. mce.2015.11.030)

9 Abbott DH, Dumesic DA, Eisner JR, Colman RJ \& Kemnitz JW. Insights into the development of polycystic ovary syndrome (PCOS) from studies of prenatally androgenized female rhesus monkeys. Trends in Endocrinology and Metabolism 19989 62-67. (doi:10.1016/ S1043-2760(98)00019-8)

10 Xita N \& Tsatsoulis A. Review: fetal programming of polycystic ovary syndrome by androgen excess: evidence from experimental, clinical, and genetic association studies. Journal of Clinical Endocrinology and Metabolism 200691 1660-1666. (doi:10.1210/jc.2005-2757)

11 Dumesic DA, Abbott DH \& Padmanabhan V. Polycystic ovary syndrome and its developmental origins. Reviews in Endocrine and Metabolic Disorders 20078 127-141. (doi:10.1007/s11154-0079046-0)

12 Recabarren SE, Smith R, Rios R, Maliqueo M, Echiburú B, Codner E, Cassorla F, Rojas P \& Sir-Petermann T. Metabolic profile in sons of women with polycystic ovary syndrome. Journal of Clinical Endocrinology and Metabolism 200893 1820-1826. (doi:10.1210/ jc.2007-2256)

13 Crisosto N, Echiburú B, Maliqueo M, Pérez V, Ladrón de Guevara A, Preisler J, Sánchez F \& Sir-Petermann T. Improvement of hyperandrogenism and hyperinsulinemia during pregnancy in women with polycystic ovary syndrome: possible effect in the ovarian follicular mass of their daughters. Fertility and Sterility 2012 97 218-224. (doi:10.1016/j.fertnstert.2011.10.002)

14 Recabarren SE, Sir-Petermann T, Rios R, Maliqueo M, Echiburú B, Smith R, Rojas-García P, Recabarren M \& Rey RA. Pituitary and testicular function in sons of women with polycystic ovary syndrome from infancy to adulthood. Journal of Clinical Endocrinology and Metabolism 200893 3318-3324. (doi:10.1210/jc.2008-0255)

15 Sir-Petermann T, Codner E, Pérez V, Echiburú B, Maliqueo M, Ladrón de Guevara A, Preisler J, Crisosto N, Sánchez F, Cassorla F, et al. Metabolic and reproductive features before and during puberty in daughters of women with polycystic ovary syndrome. Journal of Clinical Endocrinology and Metabolism 200994 1923-1930. (doi:10.1210/jc.2008-2836)

16 Ferriman D \& Purdie AW. The inheritance of polycystic ovarian disease and a possible relationship to premature balding. Clinical Endocrinology 197911 291-300. (doi:10.1111/j.1365-2265.1979. tb03077.x)

17 Cohen PN, Givens JR, Wiser WL, Wilroy RS, Summitt RL, Coleman SA \& Andersen RN. Polycystic ovarian disease, maturation arrest of spermiogenesis, and Klinefelter's syndrome in siblings of a family with familial hirsutism. Fertility and Sterility 197526 1228-1238. (doi:10.1016/S0015-0282(16)41539-6) 
18 Legro RS, Kunselman AR, Demers L, Wang SC, Bentley-Lewis R \& Dunaif A. Elevated dehydroepiandrosterone sulfate levels as the reproductive phenotype in the brothers of women with polycystic ovary syndrome. Journal of Clinical Endocrinology and Metabolism 2002 87 2134-2138. (doi:10.1210/jcem.87.5.8387)

19 Petermann TS, Cartes A, Maliqueo M, Vantman D, Gutiérrez C, Toloza H, Echiburú B \& Recabarren SE. Patterns of hormonal response to the GnRH agonist leuprolide in brothers of women with polycystic ovary syndrome: a pilot study. Human Reproduction 2004 19 2742-2747. (doi:10.1093/humrep/deh512)

20 Abreu AP \& Kaiser UB. Pubertal development and regulation. Lancet Diabetes and Endocrinology 20164 254-264. (doi:10.1016/S22138587(15)00418-0)

21 Zawadski JK \& Dunaif A. Diagnostic criteria for polycystic ovary syndrome: towards a rational approach. In Polycystic Ovary Syndrome, pp 59-69. Eds A Dunaif, JR Givens, FP Haseltine \& GE Merriam. Oxford, UK: Blackwell, 1992.

22 Adams J, Polson DW \& Franks S. Prevalence of polycystic ovaries in women with anovulation and idiopathic hirsutism. BMJ 1986293 355-359. (doi:10.1136/bmj.293.6543.355)

23 Ogden CL, Kuczmarski RJ, Flegal KM, Mei Z, Guo S, Wei R, Grummer-Strawn LM, Curtin LR, Roche AF \& Johnson CL. Centers for disease control and prevention 2000 growth charts for the United States: improvements to the 1977 National Center for Health Statistics version. Pediatrics 2002109 45-60. (doi:10.1542/ peds.109.1.45)

24 Youlton R \& Valenzuela C. Growth patterns in height and weight in children aged 0 to 17 years and cranial circumference in children aged 0 to 2 years from medium-high and high socioeconomic status in Santiago. Comparison with growth in children from medium-low and low status in the Northern area of Santiago. Revista Chilena de Pediatría 1990 1-22.
25 American Diabetes Association. Diagnosis and classification of diabetes mellitus. Diabetes Care 201437 (Supplement 1) S81-S90. (doi:10.2337/dc14-S081)

26 Matthews, DR, Hosker JP, Rudenski AS, Naylor BA, Treacher DF \& Turner RC. Homeostasis model assessment: insulin resistance and beta-cell function from fasting plasma glucose and insulin concentrations in man. Diabetologia 198528 412-419. (doi:10.1007/ BF00280883)

27 Matsuda M \& DeFronzo RA. Insulin sensitivity indices obtained from oral glucose tolerance testing: comparison with the euglycemic insulin clamp. Diabetes Care 199922 1462-1470. (doi:10.2337/ diacare.22.9.1462)

28 Qi Q, Hua S, Perreira KM, Cai J, Van Horn L, Schneiderman N, Thyagarajan B, Delamater AM, Kaplan RC \& Isasi CR. Sex differences in associations of adiposity measures and insulin resistance in US Hispanic/Latino youth: the Hispanic Community Children's Health Study/Study of Latino Youth (SOL Youth). Journal of Clinical Endocrinology and Metabolism 2017102 185-194. (doi:10.1210/ jc.2016-2279)

29 Barja S, Barrios X, Arnaiz P, Domínguez A, Villarroel L, Castillo O, Farías M, Ferreccio C \& Mardones F. Blood lipids in Chilean children 10-14 years of age. Nutrición Hospitalaria 201328 719-725. (doi:10.3305/nh.2013.28.3.3659)

30 Carmina E, Koyama T, Chang L, Stanczyk FZ \& Lobo RA. Does ethnicity influence the prevalence of adrenal hyperandrogenism and insulin resistance in polycystic ovary syndrome? American Journal of Obstetrics and Gynecology 1992167 1807-1812. (doi:10.1016/00029378(92)91779-A)

31 Hoffman DI, Klove K \& Lobo RA. The prevalence and significance of elevated dehydroepiandrosterone sulfate levels in anovulatory women. Fertility and Sterility 198442 76-81. (doi:10.1016/S00150282(16)47961-6)

Received in final form 30 August 2017

Accepted 14 September 2017

Accepted Preprint published online 14 September 2017 http://www.endocrineconnections.org DOI: 10.1530/EC-17-0218

() 2017 The authors Published by Bioscientifica Ltd

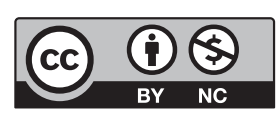

This work is licensed under a Creative Commons Attribution-NonCommercial 4.0 International License. 